\title{
3D Elastography Using Freehand Ultrasound
}

\author{
Joel Lindop, Graham Treece, Andrew Gee, and Richard Prager \\ Department of Engineering, University of Cambridge, Trumpington Street, \\ Cambridge, UK, CB2 1PZ, jel35,gmt11, ahg, rwp@eng.cam.ac.uk
}

\begin{abstract}
We present a novel technique for 3D elastography using freehand ultrasound. The scan is straightforward to perform, requiring just a single sweep over the area of interest with an unmodified $2 \mathrm{D}$ probe. The 3D elastogram is constructed in real time and can be visualised immediately following the sweep. Results are presented for a jelly phantom containing a hard inclusion.
\end{abstract}

\section{Introduction}

The term "elastography" refers to the imaging of tissue stiffness. One way of performing elastography is by ultrasonic strain imaging, whereby a sequence of ultrasound images is used to quantify tissue deformation under applied pressure. The clinical motivation for this sort of imaging is well rehearsed: hard inclusions, such as certain types of tumour, are often far easier to detect in elastograms than in conventional B-mode images. Manufacturers of ultrasound equipment are beginning to offer 2D elastography on their top-end machines. 3D elastography has been reported using a mechanically swept 3D probe [1]. Here, we present an alternative approach to 3D elastography using an unmodified $2 \mathrm{D}$ probe and a freehand scanning protocol. To the best of our knowledge, this is the first time such a system has been reported in the literature.

\section{Freehand 3D Elastography}

There are several ways to apply the time-varying pressure required for ultrasonic strain imaging. For instance, the probe can be modified to incorporate a mechanical vibrator. Here, we adopt the simplest approach whereby the clinician applies the pressure manually. For 2D elastography, the clinician holds the probe over the area of interest and applies a varying contact pressure. Pesavento et al [2] describe how the resulting radio frequency $(\mathrm{RF})$ image sequence can be analysed in real time to produce a set of $2 \mathrm{D}$ elastograms showing axial stiffness.

For our 3D system, we use a $5-10 \mathrm{MHz}$ linear array probe connected to a Dynamic Imaging Diasus ultrasound machine. The RF echo signal is digitised at $67 \mathrm{MHz}, 30$ frames per second using a Gage CompuScope 14100 analogue to digital converter. Sequential frames are compared to produce 2D elastograms in real time using Pesavento's phase root seeking algorithm 2]. The position and orientation of the probe are tracked using a Northern Digital Polaris optical position sensor: the 2D elastograms can therefore be located in space and reconstructed in 3D using the Stradx freehand 3D ultrasound system. The 3D point precision of this system has been measured at around $0.6 \mathrm{~mm} 3$. 
The freehand scanning protocol for 3D elastography is surprisingly simple: it transpires that a single sweep is sufficient. Compared with the axial resolution, the elevational and lateral resolutions are poor. Given the high acquisition rate, speckle decorrelation between neighbouring frames is due almost entirely to axial strain and not elevational or lateral movement. Thus, meaningful 2D elastograms can be constructed between neighbouring frames, even though the motion is predominantly elevational and not axial. The operator need make no special effort to vary the contact pressure: the system is sufficiently sensitive to detect the involuntary variations implicit in a freehand scan. Figure 1 shows 3D elastography of a hard inclusion (an olive) embedded in a jelly phantom.

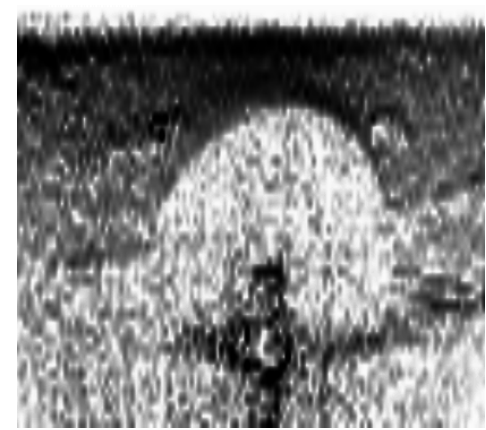

(a)

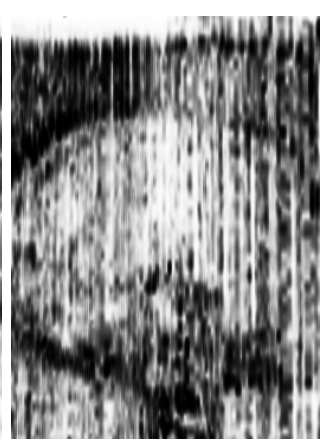

(b)

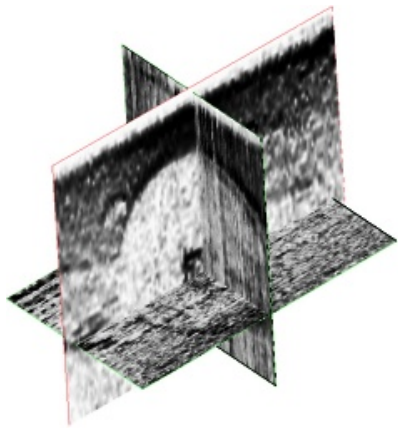

(c)

Fig. 1. 3D elastography of jelly/olive phantom. (a) is a 2D elastogram, (b) is a perpendicular reslice through the elastograms, (c) shows an elastogram and two such reslices.

\section{Conclusions}

We have presented a novel system for freehand 3D elastography. The scanning protocol is simple and the elastograms are constructed in real time using a standard PC and unmodified freehand 3D ultrasound equipment.

\section{References}

1. Lorenz, A., Pesavento, A., Pesavento, M., Ermert, H.: Three-dimensional strain imaging and related strain artifacts using an ultrasonic 3D abdominal probe. In: Proceedings of the 1999 IEEE Ultrasonics Symposium. Volume 2. (1999) 1657-1660

2. Pesavento, A., Perrey, C., Krueger, M., H., E.: A time efficient and accurate strain estimation concept for ultrasonic elastography using iterative phase zero estimation. IEEE T Ultrason Ferr 46 (1999) 1057-1067

3. Treece, G.M., Gee, A.H., Prager, R.W., Cash, C.J.C., Berman, L.: High definition freehand 3D ultrasound. Ultrasound Med Biol 29 (2003) 529-546 